ISSN: 2591-7994

\title{
Pathogenesis and treatment status of adenomyosis complicated with infertility.
}

\section{Hua Wang, Chun-hua Cao, Yan Chen, Saili Wang, Qin Wang*}

Taizhou Hospital of Traditional Chinese Medicine, Affiliated to Nanjing University of Traditional Chinese Medicine, Taizhou, China

\section{Retraction Note:}

The article entitled "Pathogenesis and treatment status of adenomyosis complicated with infertility" has been accepted for publication in the Journal of Gynecology and Reproductive Endocrinology considering the statements provided in the article as personal opinion of the author which was found not having any conflict or biasness towards anything. As the article was a perspective one, information provided by the author was considered as an opinion to be expressed through publication.

Soon after the publication of the paper, we witnessed some serious concerns and many of them argued that the paper is a personal perspective and had not discussed any relevant ethical issue considered under the journal scope. Moreover, the paper is neither innovative nor thought provoking.

Publisher took decision to make the article online solely based on the reviewers suggestion which considered the article not but a personal opinion of the author. However, it is found that the article has some unavoidable mistakes and issues, therefore, being retracted from the journal.

\section{Review Comments on this manuscript:}

This article shows the importance and the needed to have validated tools to assess. Interesting topic about the perception of a worker has in this work place.

Work done is apt

Research done is authentic

Bibliography is accurate and as per norms

Tables are consistent with the results

Conclusion matches with the objectives

Good job and great explanation of the validation process.

To be accepted for further publication 